\title{
X Congreso Ibérico de Agroingeniería

\section{Comprobación de un sistema de aplicación variable basado en mapas de vegetación obtenidos con un vehículo aéreo no tripulado (UAV)}

\author{
J. Campos ${ }^{1}$, M. Gallart ${ }^{1}$, J. Llop ${ }^{1}$, R. Salcedo ${ }^{1}$, P. Ortega ${ }^{1}$, E. Armengol ${ }^{1}$, E. Gil ${ }^{1}$ \\ 1 Departamento de Ingeniería Agroalimentaria y Biotecnología, Universidad Politécnica de Cataluña, \\ Esteve Terradas, 8 - 08860 - Castelldefels, Barcelona (España); javier.campos@upc.edu.
}

\begin{abstract}
Resumen: Las características de la vegetación son un aspecto fundamental a tener en cuenta para determinar el volumen de aplicación de productos fitosanitarios. El alto grado de variabilidad intraparcelaria ha aumentado la dificultad de obtener soluciones generales para la determinación del volumen óptimo de aplicación. En este sentido, actualmente se está trabajando con sistemas de aplicación variable a tiempo real considerando las características de la vegetación. Este estudio presenta el proceso de pulverización variable en base a mapas de prescripción, desde la creación del mapa de prescripción hasta la obtención del mapa de aplicación real. Los ensayos se realizaron en Torrelavit (Barcelona) en una parcela de viña de 2.21 ha de la variedad Chardonnay. El mapa de prescripción se obtuvo combinando las imágenes multiespectrales obtenidas con un UAV con la información proporcionada por DOSAVIÑA $^{\circledR}$. Este mapa de prescripción fue cargado en un prototipo de aplicación variable, obteniendo un mapa de aplicación real después del proceso de aplicación. Los resultados obtenidos muestran la posibilidad de ahorra alrededor de un $20 \%$ la cantidad de agua gastada y la cantidad de materia activa respecto a un tratamiento convencional de productos fitosanitarios.
\end{abstract}

Palabras clave: Mapa de prescripción, mapa de aplicación real, Dosaviña ${ }^{\circledR}$

\section{Introducción}

La aplicación de fitosanitarios en base a la vegetación ha sido ampliamente estudiada [p. ej. $1,2,3]$. En todos los casos, el objetivo principal ha sido la adaptación de la cantidad total del producto fitosanitario a las características del cultivo. El alto grado de variabilidad en las características de la vegetación ha aumentado la dificultad de obtener soluciones generales bien adaptadas a todos los cultivos y situaciones. En este sentido, la aplicación variable es capaz de reducir el efecto de la variabilidad intraparcelaria, ajustando el volumen de caldo a las características de la vegetación en cada momento.

Actualmente se pueden diferenciar dos tipos de tecnología de aplicación variable: unas están basadas en la aplicación en tiempo real a partir de información obtenida por sensores; y otras se fundamentan en mapas de prescripción.

La tecnología de aplicación variable basada en sensores en tiempo real elimina la necesidad del conocimiento previo del cultivo, ya que se aplica en función de lo que los sensores determinen en cada momento [p.ej. 4, 5].

Por otro lado, la tecnología de aplicación variable basada en mapas de prescripción presenta la necesidad de realizar previamente a la aplicación el estudio del cultivo, ya sea mediante datos recopilados manualmente en campo o mediante teledetección. En este sentido, [6] evaluaron la capacidad de un pulverizador hidráulico modificado para la 


\section{CONGRESO IBÉRICO DE AGROINGENIERÍA \\ X CONGRESSO IBÉRICO DE AGROENGENHARIA \\ 3 - 6 septiembre 2019, Huesca - España}

aplicación variable de herbicida basada en diferentes mapas de prescripción con el volumen de aplicación prescrito al controlador del pulverizador concluyendo que el sistema era capaz de combatir los rodales de malas hierbas en cultivos de maíz y soja. En otro estudio, [7] desarrollaron un prototipo de aplicación variable en base a mapas, obtenidos de imágenes espectrales aéreas, para combatir las malas hierbas en el cultivo del arándano. Cabe destacar que la tecnología de aplicación variable de productos fitosanitarios en base a mapas de prescripción es ampliamente utilizada comercialmente en cultivos extensivos, pero no en cultivos 3D, donde la tecnología es muy incipiente.

Los objetivos de este trabajo fueron el desarrollo y puesta a punto de un sistema de aplicación variable de productos fitosanitarios en base a mapas de prescripción realizados a partir de imágines espectrales obtenidas mediante UAV para viña, la determinación de la precisión del sistema, y el cálculo de los ahorros potenciales respecto a una aplicación convencional.

\section{Materiales y métodos}

\subsection{Ubicación de los ensayos}

Los ensayos se realizaron en una parcela de viña de 2.21 ha situada en la comarca del Penedès (Barcelona) (X: 392194 m, Y: 4588000 m, ETRS89 UTM31). Se trata de una parcela de la variedad Cabernet Sauvignon con un sistema de formación Guyot Doble y un marco de plantación de $1.2 \mathrm{~m}$ entre plantas y $2.2 \mathrm{~m}$ entre hileras. Los ensayos se llevaron a cabo durante el estado fenológico $\mathrm{BBCH} 77-79$ [8].

\subsection{Adquisición de datos para la caracterización de la vegetación}

La parcela se sobrevoló con un vehículo aéreo no tripulado (UAV) cargado con una cámara multiespectral RedEGDE (Micasense, Seattle, USA), equipada con 5 bandas espectrales (R, G, B, RedEdge y NIR). El vuelo se realizó a $95 \mathrm{~m}$ sobre el nivel del suelo y se planificó para realizar un $80 \%$ de solape frontal y un $60 \%$ de solape lateral.

\subsection{Pulverizador adaptado para la aplicación variable}

El sistema de aplicación variable de instaló en un pulverizador hidroneumático (Saher, Barcelona) con un tanque de $1000 \mathrm{~L}$ y con un ventilador axial de $800 \mathrm{~mm}$ de diámetro. Con el fin de seguir el mapa de prescripción, se equipó el pulverizador con a) un sensor de presión GEMS 1200 series (Gems Sensors \& Controls, Plainville, USA) para monitorizar en todo momento la presión real y poder reajustarla en función de lo establecido por el mapa de prescripción, b) un sistema de control electrónico (Estel S.L., Barcelona) compuesto por un receptor GNSS con una frecuencia de $1 \mathrm{~Hz}$, una pantalla táctil y un controlador de secciones. La función del sistema de control eléctrico fue posicionar al pulverizador en la parcela mediante el receptor GNSS, determinar el volumen de aplicación prescrito en función del mapa previamente cargado y modificar la presión de trabajo para ajustarse al caudal de las boquillas deseado.

\subsection{Sistema de ayuda a la decisión óptima del volumen de aplicación}

Se utilizó el sistema de ayuda a la decisión (DSS) DOSAVIÑA ${ }^{\circledR}$ [9] basado en el método "leaf wall area" (LWA) [10] para la determinación del volumen de aplicación óptimo a pulverizar basado en las características estructurales de la vegetación.

\subsection{Metodología del proceso}

El proceso completo de la aplicación variable basada en mapas se muestra en la Figura 1. Se obtuvo una ortofoto a partir de las imágenes espectrales adquiridas con la cámara multiespectral embarcada en el UAV. La ortofoto fue calibrada radiométricamente utilizando cuatro estándares 


\section{CONGRESO IBÉRICO DE AGROINGENIERÍA \\ X CONGRESSO IBÉRICO DE AGROENGENHARIA \\ 3 - 6 septiembre 2019, Huesca - España}

de escalas de grises (22, 32, 44 y 51\% de reflectancia de escala de grises) colocados en el suelo durante el vuelo, con el fin de transformar valores digitales de escala de grises de 12 bits en valores de reflectancia. A partir de estos nuevos valores se calculó el índice de vegetación diferencial normalizado (NDVI) [11] (Ecuación 1).

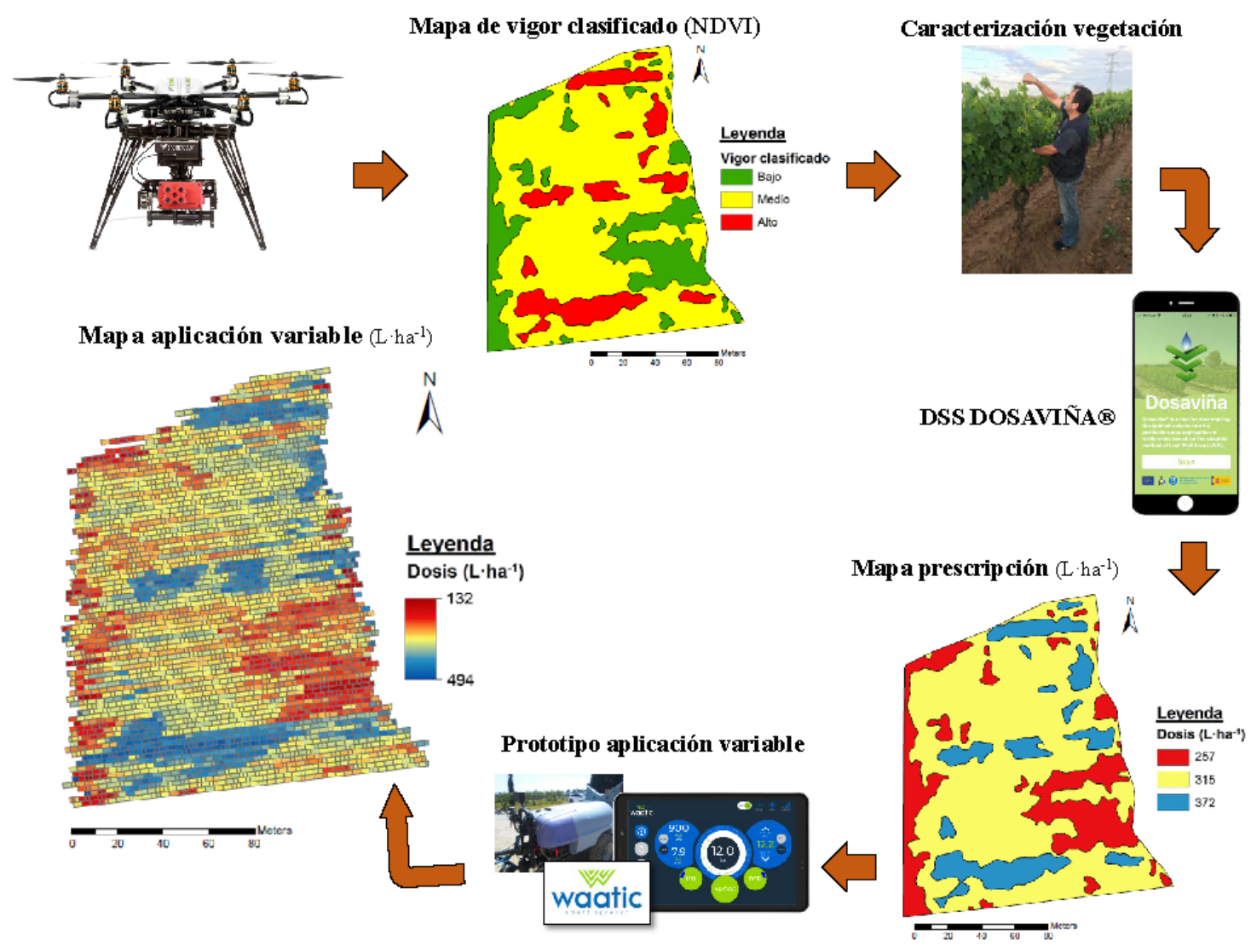

Figura 1. Esquema del proceso: desde el UAV hasta el mapa de aplicación real

$$
\mathrm{NDVI}=\frac{\mathrm{NIR}-\mathrm{RED}}{\mathrm{NIR}+\mathrm{RED}}
$$

Debido al crecimiento en líneas de la viña, la imagen fue segmentada por un umbral de NDVI con el fin de eliminar malas hierbas, sombras y suelo entre líneas de cultivo. Una vez aplicado el umbral de NDVI, se realizó una interpolación por el método de distancia inversa ponderada (IDW) para obtener un mapa continuo de NDVI. Finalmente, se realizó un procesado de este mapa con el objetivo de agrupar los valores de NDVI en tres niveles, que luego se suavizaron mediante un filtrado por el vecino más próximo para obtener el mapa de vigor clasificado final. El proceso completo se realizó utilizando el software QGIS [12]. Una vez creado el mapa de vigor clasificado en tres zonas de vigor diferenciadas (bajo, medio y alto), fueron localizadas en la parcela. Para obtener el volumen de aplicación óptimo mediante DOSAVIÑA ${ }^{\circledR}$ [13], se realizaron 15 caracterizaciones manuales de la vegetación en cada una de las zonas de vigor diferenciadas (Tabla 1). 
X CONGRESO IBÉRICO DE AGROINGENIERÍA

X CONGRESSO IBÉRICO DE AGROENGENHARIA

3 - 6 septiembre 2019, Huesca - España

Tabla 1. Caracterización de la vegetación y volúmenes obtenidos mediante DOSAVIÑA®

\begin{tabular}{ccccc}
\hline Vigor & $\begin{array}{c}\text { Altura } \\
\text { vegetación } \mathbf{( m )}\end{array}$ & $\begin{array}{c}\text { Anchura } \\
\text { vegetación } \mathbf{( m )}\end{array}$ & $\begin{array}{c}\text { LWA }^{*} \\
\left(\mathbf{m}^{\mathbf{2}} \text { vegetación }^{*} \mathbf{h a}^{-\mathbf{1}}\right)\end{array}$ & $\begin{array}{c}\text { Volumen (L·ha- } \\
\mathbf{1})\end{array}$ \\
\hline Bajo & 0.92 & 0.37 & 8363.6 & 257 \\
Medio & 1.11 & 0.44 & 10045.5 & 315 \\
Alto & 1.27 & 0.48 & 11545.5 & 372 \\
\hline
\end{tabular}

*LWA calculado para una distancia entre hileras de $2.2 \mathrm{~m}$

Los valores obtenidos se introdujeron en el mapa de vigor utilizando el software QGIS [12] para obtener el mapa de prescripción. El mapa de prescripción fue cargado mediante USB a la pantalla táctil instalada previamente en el pulverizador. Además, se cargó en el sistema información relativa a los parámetros de trabajo para cada zona de vigor (Tabla 2). El número y el tipo de boquilla se mantuvieron constantes en todos los casos, utilizando boquillas de cono hueco Albuz ATR (Albuz Saint-Govain, Evreux, Francia).

Tabla 2. Condiciones de aplicación para cada una de las zonas de vigor

\begin{tabular}{cccccc}
\hline Vigor & $\begin{array}{c}\text { Volumen } \\
\left(\mathbf{L} \cdot \mathbf{h a}^{-\mathbf{1}} \mathbf{)}\right.\end{array}$ & $\begin{array}{c}\text { Tipo de } \\
\text { boquilla }\end{array}$ & $\begin{array}{c}\text { No de } \\
\text { boquillas }\end{array}$ & $\begin{array}{c}\text { Presión } \\
\text { (bar) }\end{array}$ & $\begin{array}{c}\text { Velocidad } \\
\mathbf{( k m} \cdot \mathbf{h}^{-1} \mathbf{)}\end{array}$ \\
\hline Bajo & 257 & ATR Amarillo & 8 & 6 & 6.8 \\
Medio & 315 & ATR Amarillo & 8 & 9.2 & 6.8 \\
Alto & 372 & ATR Amarillo & 8 & 12.8 & 6.8 \\
\hline
\end{tabular}

Una vez cargados los parámetros en la pantalla táctil, se inició la pulverización. Desde el inicio, el sistema registró con una frecuencia de 1 segundo la posición del pulverizador en la parcela, el volumen de aplicación real y la presión de trabajo ajustada. Al final, el sistema generó un mapa de aplicación real que se descargó desde la pantalla táctil mediante USB.

\subsection{Comparación del mapa de prescripción con el mapa de aplicación real}

Con el fin de evaluar el sistema de aplicación variable utilizado, se compararon el mapa de prescripción y el mapa de aplicación real utilizando el software QGIS [12]. Se generaron aleatoriamente 44,000 puntos aproximadamente para realizar la comparación. Cada uno de estos puntos georeferenciados adquirió un valor prescrito "r" y un valor real "p". Para cada valor del valor esperado "r", se asignaron 11 intervalos de tolerancia (cada uno de ellos representaba un aumento del 5\% en comparación con el anterior, desde el $0 \%$ hasta el 50\% de desviación). Se determinó si el valor de $\mathrm{p}$ estaba dentro del rango calculado [r-i, r+i]. Una vez comparados todos los puntos, también se calculó el porcentaje de coincidencia entre los valores esperados y los reales. Finalmente, para visualizar el nivel de precisión del mapa de aplicación real, se realizó una interpolación siguiendo el método de distancia inversa ponderada (IDW).

\section{Resultados y discusión}

\subsection{Mapas generados}

En este subapartado se muestran los mapas generados durante todo el proceso de aplicación variable. La secuencia de mapas generados es la siguiente: (1) mapa de NDVI, (2) mapa de vigor clasificado, (3) mapa de prescripción y (4) mapa de aplicación real (Fig. 2). 


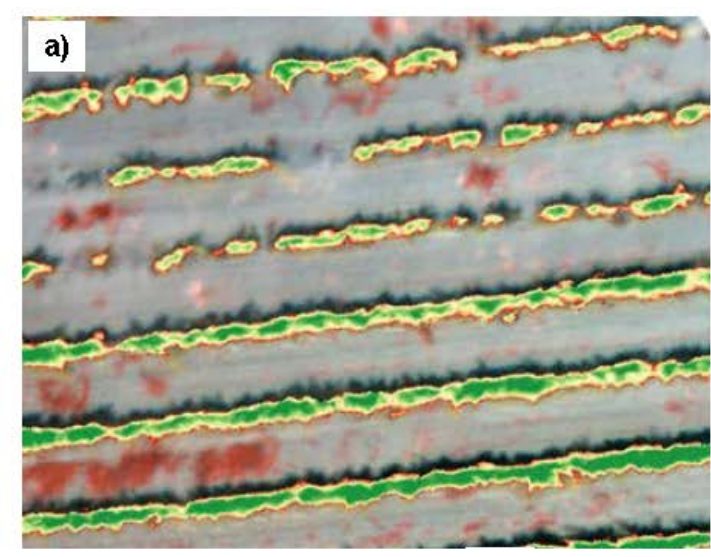

c)

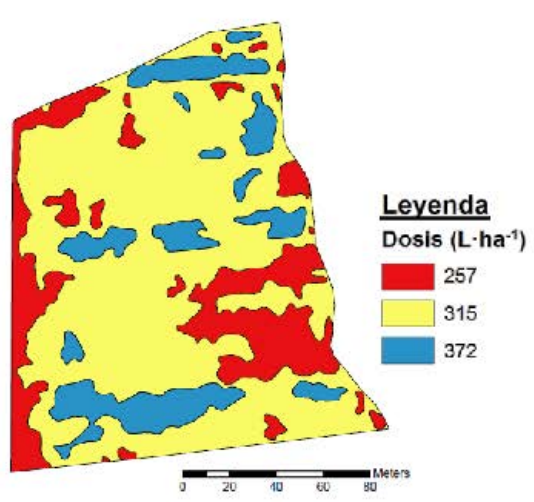

b)

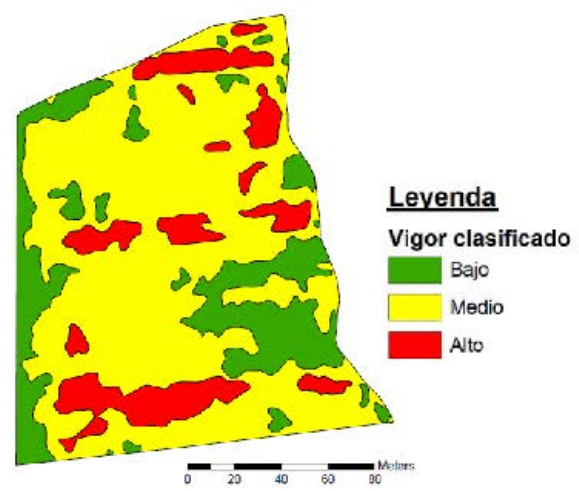

d)

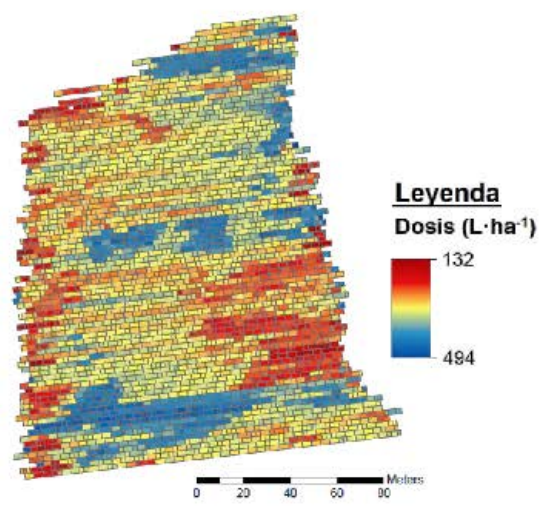

Figura 2. a) Mapa de NDVI puro; b) Mapa de vigor clasificado; c) Mapa de prescripción; d) Mapa de aplicación real

\section{Mapa de NDVI}

Con las imágenes obtenidas con la cámara multiespectral embarcada en el UAV, se generó el mapa de NDVI (Figura 2a). Este mapa nos indica el estado de la vegetación

\section{Mapa de vigor clasificado}

Una vez obtenido el mapa de NDVI, se procesó para obtener tres zonas diferenciadas en la parcela. El mapa de vigor obtenido se muestra en la Figura $2 b$. Este mapa nos determina zonas diferenciadas de vigor. Teniendo en cuenta la superficie total de la parcela (2.21ha), las zonas de vigor se han distribuido de la siguiente manera: la zona de bajo vigor (zona verde) representa un $20 \%$ de la superficie total, la zona de vigor medio (zona amarilla) representa un $66 \%$ de la superficie total y la zona de vigor alto (zona naranja) representa un $14 \%$ de la superficie total de la parcela.

\section{Mapa de prescripción}

La Tabla 1 muestra los resultados obtenidos para cada una de las zonas caracterizadas manualmente. Los valores obtenidos fueron $257 \mathrm{~L} \cdot \mathrm{ha}^{-1}$ para el vigor bajo, $315 \mathrm{~L} \cdot \mathrm{ha}^{-1}$ para el vigor medio y $372 \mathrm{~L} \cdot \mathrm{ha}^{-1}$ para el vigor alto. A partir de estos valores se generó el mapa de prescripción (Figura 2c).

\section{Mapa de aplicación real}

Durante el proceso de aplicación el sistema de aplicación variable guardó información respecto a la posición del pulverizador en la parcela, el volumen de aplicación real y la presión real de 


\section{CONGRESO IBÉRICO DE AGROINGENIERÍA \\ X CONGRESSO IBÉRICO DE AGROENGENHARIA \\ 3- 6 septiembre 2019, Huesca - España}

trabajo, con una frecuencia de $1 \mathrm{~s}$. Al final, el sistema generó un mapa de aplicación real (Figura 2d) que se descargó desde la pantalla táctil instalada en el pulverizador.

\subsection{Precisión del sistema}

Para cuantificar la correspondencia entre el mapa de prescripción y el mapa de aplicación real, se establecieron diferentes umbrales desde $0 \%$ hasta $50 \%$ de tolerancia. El umbral más restrictivo $(0 \%)$ determinó el porcentaje de puntos en los que no había diferencia entre el mapa de prescripción y el mapa de aplicación real. En este caso, sólo el 1,3\% de los puntos se clasificaron exitosamente. En el extremo opuesto, la tolerancia más alta (50\%) cuantificó el porcentaje de puntos en los que se detectaron variaciones de $\pm 50 \%$ del volumen aplicado. En este caso, el 99, $8 \%$ de los puntos se clasificaron exitosamente. Suponiendo que desde un punto de vista práctico se pudiera permitir una tolerancia de $\pm 10 \%$, el sistema clasificó el $75 \%$ de los puntos exitosos. La Figura 3 muestra la precisión distribuida alrededor de la parcela, clasificada según el nivel de umbral establecido. Las zonas oscuras de los mapas indican las zonas en las que la precisión del sistema superó los umbrales establecidos. El porcentaje principal de zonas oscuras corresponde a las zonas de transición, donde se ordenó al pulverizador que modificara la presión de trabajo. Como muestran los resultados, en términos de comparación entre el mapa actual y el mapa de prescripción, el método de aplicación de dosis variable obtuvo una buena precisión.

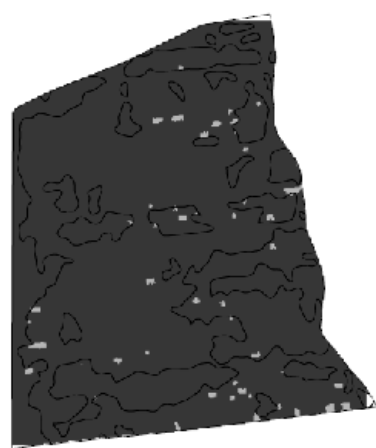

$0 \%$ tolerancia

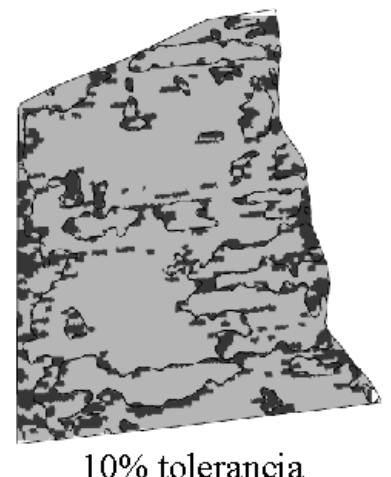

$10 \%$ tolerancia

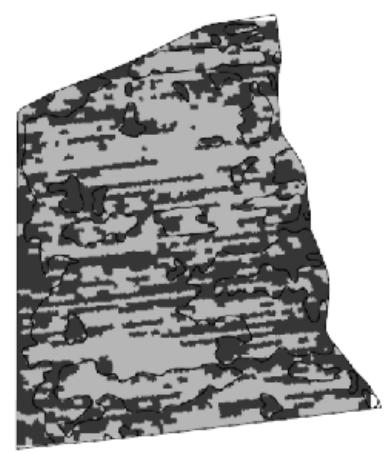

$5 \%$ tolerancia

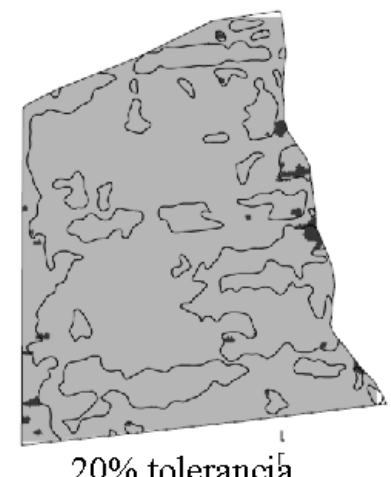

$20 \%$ tolerancia

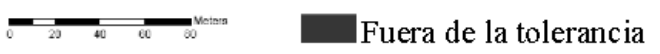

Dentro de la tolerancia

Figura 3. Distribución de la precisión a lo largo de la parcela

\subsection{Cuantificación de ahorros}

El proceso de aplicación variable basado en mapas se comparó con una aplicación convencional basado en un volumen constante de $380 \mathrm{~L} \cdot \mathrm{ha}^{-1}$ ha, volumen normalmente utilizado por los agricultores de la zona. Para estos dos casos, se calculó la cantidad de agua utilizada, el 


\section{CONGRESO IBÉRICO DE AGROINGENIERÍA \\ X CONGRESSO IBÉRICO DE AGROENGENHARIA \\ 3 - 6 septiembre 2019, Huesca - España}

tiempo total de aplicación suponiendo 45 minutos para el llenado de la máquina y la cantidad hipotética de materia activa (m.a.) utilizada, suponiendo una concentración de cobre de 0,4\% (400 $\mathrm{g} \cdot \mathrm{hL}^{-1}$ ) (Tabla 3). Los resultados muestran un efecto positivo del uso de la aplicación de dosis variable. A pesar de no obtener un ahorro en el tiempo empleado, la cantidad de líquido consumido y la cantidad de materia activa se redujeron en un $18.4 \%$ utilizando la aplicación variable de productos fitosanitarios basada en mapas.

Tabla 3. Comparación entre una aplicación convencional y una aplicación variable (VRA)

\begin{tabular}{ccccccccc}
\hline & $\begin{array}{c}\text { Vol. (L·ha- } \\
\text { () }\end{array}$ & $\begin{array}{c}\text { Vol. } \\
\text { aplicado } \\
\text { (L) }\end{array}$ & $\begin{array}{c}\text { Ahorro } \\
\text { vol. (\%) }\end{array}$ & $\begin{array}{c}\text { No } \\
\text { llenados }\end{array}$ & $\begin{array}{c}\text { Tiempo } \\
\text { (h) }\end{array}$ & $\begin{array}{c}\text { Ahorro } \\
\text { tiempo } \\
\mathbf{( \% )}\end{array}$ & $\begin{array}{c}\text { m.a. } \\
\mathbf{( k g )}\end{array}$ & $\begin{array}{c}\text { Ahorro } \\
\text { m.a. } \\
\mathbf{( k g})\end{array}$ \\
\hline Conv & 380 & 808 & 0 & 1 & 6.8 & 0 & 3.23 & 0 \\
VRA & $257 / 315 / 372$ & 659 & 18.4 & 1 & 6.8 & 0 & 2.64 & 18.4 \\
\hline
\end{tabular}

\section{Conclusiones}

En el presente trabajo se ha puesto a prueba un prototipo de aplicación variable de productos fitosanitarios en base a mapas de prescripción. Los resultados mostrados anteriormente ponen de manifiesto algunos hechos importantes: 1)El sistema es capaz de cargar y leer un mapa de prescripción y modificar la presión de trabajo según su posición en la parcela; 2) El mapa de vigor clasificado se transformó de manera fácil a un mapa de prescripción teniendo en cuenta las características estructurales de la vegetación; 3) Se ha obtenido una precisión de aproximadamente el $80 \%$ con el sistema, asumiendo una tolerancia del 10\% de desviación; 4) El uso de mapas de vigor clasificados combinados con el uso del DOSAVIÑA ${ }^{\circledR}$ para determinar el volumen óptimo de aplicación puede reducir alrededor de un $20 \%$ la cantidad de agua gastada y la cantidad de materia activa, siempre y cuando se mantenga la concentración.

\section{Agradecimientos}

Este trabajo se ha financiado parcialmente por los “Ajuts a les activitats de demostració (operació 01.02.01 de Transferència Tecnològica del Programa de desenvolupament rural de Catalunya 2014-2020)" y la beca FI-AGAUR. La validación del Dosaviña ha sido desarrollada en el marco del proyecto LIFE PERFECT project: Pesticide Reduction using Friendly and Environmentally Controlled Technologies (LIFE17 ENV/ES/000205).

\section{Referencias}

1. Furness G.O. Distance calibration and a new pesticide label format for fruit trees and grapevines in Australia. Proceedings of the Seventh Workshop on Spray Application Techniques in Fruit Growing. Cuneo, Italy, 2003, pp. 293-303.

2. Pergher G., Petris R. Pesticide dose adjustment in vineyard spraying and potential for dose reduction. Agricultural Engineering International: the CIGR Ejournal. Manuscript ALNARP 08 011. 2008, Vol. X.

3. Gil E., Gallart M., Llorens J., Llop J., Bayer T., Carvalho C. Spray adjustments based on LWA concept in vineyard. Relationship between canopy and coverage for different application settings. International Advances in Pesticide Applications. Aspects of Applied Biology, 2014, 122 25-32.

4. Giles D.K., Delwiche M.J., Dodd, R.B. Sprayer control by sensing orchard crop characteristics: orchard architecture and spray liquid savings. J. Agric. Eng. Res, 1989, 43, 271-289.

5. Gil E., Escolà A., Rosell J.R., Planas S., Val, L. Variable rate application of plant protection products in vineyard using ultrasonic sensors. Crop Protection, 2007, 26, 1287-1297.

6. Vogel J.W., Wolf R.E., Dille A. Evaluation of a Variable Rate Application System for Site-Specific Weed Management. ASAE. Paper No. 051120, St Joseph, MI, USA, 2008.

7. Michaud M., Watts K.C., Percival D.C., Wilkie K.I. Precision pesticide delivery based on aerial spectral imaging. Canadian Journal of Biosystems Engineering. 2008, 50, 2.9-2.15 
X CONGRESO IBÉRICO DE AGROINGENIERÍA

X CONGRESSO IBÉRICO DE AGROENGENHARIA

3- 6 septiembre 2019, Huesca - España

8. Meier U. BBCH-Monograph. Growth stages of plants - Entwicklungsstadien von Pflanzen - Estadios de las plantas - Développement des Plantes. Blackwell Wissenschaftsverlag, Berlin und Wien. 1997, $622 \mathrm{p}$

9. UMA-UPC. 2018. DOSAVIÑA®. Decision Support System for determining the optimal volume rate in vineyard. URL: https://dosavina.upc.edu (last accessed August 2018)

10. Walklate P., Cross J. An examination of Leaf-Wall-Area dose expression. Crop Protection, 2012, 35, 132134.

11. Rouse J.W., Haas R.H., Schell J.A., Deering D.W. Monitoring vegetation systems in the Great Plains with ERTS, Third ERTS Symposium, NASA SP-351 I, 1973, 309-317.

12. QGIS Development Team, 2012. QGIS Geographic Information System. Open Source Geospatial Foundation Project. https://qgis.org.

13. Gil E., Llorens J., Landers A., Llop J., Giralt L. Field validation of DOSAVIÑA, a decision support system to determine the optimal volume rate for pesticide application in vineyards. European Journal of Agronomy, 2011, 35(1), 33-46; doi:10.1016/j.eja.2011.03.005 